\title{
Certainty Factor Estimation Using Petri Neural Net for HSLA Steel
}

\author{
S. DATTA and M. K. BANERJEE
}

Department of Metallurgy, B. E. College (a Deemed University), Howrah 711103 India. E-mail: sdatta@matsc.becs.ac.in

(Received on June 29, 2004; accepted in final form on September 10, 2004)

\begin{abstract}
An unsupervised learning technique and an associative memory have been used for encoding weights by a special type of Petri network named Petri neural net for modelling the influence of alloying elements on the final property of the high strength low alloy steel. The combined effects of alloying elements for different strengthening mechanisms is predicted when weights and threshold values are chosen on the basis of metallurgical understanding. The technique is found to be effective to create an associative memory of input-output relations in unknown data sets so that the same can be subsequently be used as a predictive tool.
\end{abstract}

KEY WORDS: high strength low alloy steel; yield strength; modelling; Petri neural net; unsupervised learning.

\section{Introduction}

It is known that the alloying elements in high strength low alloy (HSLA) steels influence the final property of the steel in a complicated manner. Different strengthening mechanisms operative in HSLA steel are mainly controlled by the alloying addition. Therefore a good knowledge of the effect of alloying elements on the structure and property of HSLA steel is necessary for designing and development of steels with target properties.

While trying to solve real world problems, knowledge plays an important role in the reasoning process of a system. Rules in a knowledgebase are generally uncertain and so the execution of a rule depends on its degree of certainty when all its antecedent clauses are present. Certainty factor $^{1)}$ of rules, are generally assigned by the human experts based on their subjective judgment and past experience. Thus, they are not free from personal error and bias. Reasoning under such circumstances quite often yields inaccurate measure in the inferences. It is known that the patterns relating the inputs and outputs in HSLA steels are qualitatively recognised by the experts in the field of metallurgy. Artificial neural network (ANN) is able to map the existing input output relationship in a much better way. Used with back propagation algorithm this technique has been found to be very useful in predicting the mechanical properties of steels, which depend on a large number of independent input variables. ${ }^{2-8)}$

Automatic estimation by unsupervised learning technique $^{9)}$ and an associative memory can be used for encoding weights. An associative memory is a special type of neural net that is used for adapting weights between neurons, where the neurons determines the output pattern when the input pattern is known. The adapted or encoded weights are thus stored in the associative memory, for subsequent use in the recall phase with new input data pattern. An effort was made earlier to predict the strength properties of
HSLA steel through Kohonen network using unsupervised learning technique. ${ }^{10)}$ For such automatic estimation a new approach has been proposed in the present paper. Here an unsupervised learning technique and an associative memory ${ }^{9,11)}$ have been used for encoding weights by a special type of Petri network $(\mathrm{PN})^{12)}$ named Petri neural net (PNN). ${ }^{13,14)} \mathrm{PN}$, which has been successfully used for knowledge representation, ${ }^{15,16)}$ has equally established its importance in learning processes and has been extended here for realization of associative memory. Effort has been made to develop a stochastic model using PNN, in which, the places and transitions are allocated as neurons. In this model, the input variables are used to predict the different strengthening mechanisms, which operate on steels to determine its final properties.

\section{Database}

The alloy chemistry and amount of deformation have been taken as input parameters, whereas yield strength (YS) is designated as the output variable. Within the alloy chemistry, the concentrations of elements like carbon, manganese, silicon, nickel and chromium are kept constant in the used database. The nominal composition of the steel is given in Table 1(a). The data ranges and mean value of the variable portion of the composition (copper, molybdenum, niobium and boron), deformation percentage and yield strength is shown in Table 1(b). The data used for the present exercise ( $\sim 100$ numbers) have been generated in the laboratories. The chemical analyses are done in atomic spectrometer. Controlled rolling has been carried out in a laboratory scale two high rolling mill. The mechanical testing has been carried out in INSTRON 4204 machine.

\section{Modelling Technique}

In PNN, initial values are assigned at the input places of 
Table 1(a). The constant portion of the composition of the steels.

\begin{tabular}{|c|c|c|c|c|}
\hline $\begin{array}{c}\mathrm{C} \\
(w t \%)\end{array}$ & $\begin{array}{c}\mathrm{Mn} \\
(w t \%)\end{array}$ & $\begin{array}{c}\mathrm{Si} \\
(w t \%)\end{array}$ & $\begin{array}{c}\mathrm{Ni} \\
(w t \%)\end{array}$ & $\begin{array}{c}\mathrm{Cr} \\
(w t \%)\end{array}$ \\
\hline 0.06 & 1.45 & 0.49 & 1.12 & 0.85 \\
\hline
\end{tabular}

Table 1(b). The range of variables.

\begin{tabular}{|c|c|c|c|c|c|c|}
\hline Variables & $\begin{array}{c}\mathrm{Cu} \\
(\mathrm{wt} \%)\end{array}$ & $\begin{array}{c}\mathrm{Mo} \\
(\mathrm{wt} \%)\end{array}$ & $\begin{array}{c}\mathrm{Nb} \\
(\mathrm{wt} \%)\end{array}$ & $\begin{array}{c}\mathrm{B} \\
(\mathrm{wt} \%)\end{array}$ & $\begin{array}{c}\text { Deformation } \\
(\%)\end{array}$ & $\begin{array}{c}\mathrm{YS} \\
(\mathrm{Mpa})\end{array}$ \\
\hline $\begin{array}{c}\text { Data } \\
\text { range }\end{array}$ & $0-2$ & $0-1$ & $0-0.1$ & $0-0.003$ & $40-80$ & $700-950$ \\
\hline Mean & 1.2 & 0.55 & 0.45 & 0.002 & 65 & 826 \\
\hline
\end{tabular}

the PNN and initial certainty factor (CF) of rules are assigned too, based on the domain expert knowledge at the links between each transition and place as connection strength or weight. The stochastic truth token (STT) of all transitions are updated synchronously. This is followed by value updation at all places, also synchronously. Finally, following the Hebbian learning principle ${ }^{17)}$ the weights are updated throughout the network. The process of updation continues till the system reaches an equilibrium state. The unsupervised learning method, which has been discussed later in this section for encoding of weights, narrates the cognitive learning process. ${ }^{18)}$ The PNN, therefore, provides reliable information storage capability, similar to the concept of long-term information storage in the brain. During the recall phase the steady-state weights are used for subsequent reasoning in problems with similar databases.

\subsection{The Encoded Model}

Before presenting the encoded model, a definition of $\mathrm{PNN}$ is given below.

A PNN is a directed bipartite graph and can be represented by

$$
\mathrm{PNN}=\{P, D, T r, N, T, T h, W, I, O\}
$$

where $P=\left\{p_{1}, p_{2}, \ldots \ldots, p_{n}\right\}$ is a finite set of places, $D=\left\{d_{1}\right.$, $\left.d_{2}, \ldots \ldots, d_{n}\right\}$ is a finite set of propositions mapped at the corresponding places, for $1 \leq i \leq n$ and $|P|=|D| ; N=\left\{n_{1}, n_{2}\right.$, ......, $\left.n_{n}\right\}$ is a finite set of beliefs, each $n_{i}$ having correspondence to each proposition $d_{i}, T r=\left\{t r_{1}, t r_{2}, \ldots \ldots ., t r_{m}\right\}$ is a finite set of transitions, where $P \cap D \cap T r=\varphi, T=\left\{t_{1}, t_{2}, \ldots \ldots\right.$. $\left.t_{m}\right\}$ is a finite set of stochastic truth token (STT) associated with each transition, $T h=\left\{t h_{1}, t h_{2} . \ldots . . . t h_{m}\right\}$ is a finite set of threshold assigned at each transition, $W=\left\{w_{1,2} \ldots \ldots\right.$, $\left.w_{i j}, \ldots . ..\right\}$ is a finite set of weight; $w_{i j}$ denotes connection strength between transition $t r_{i}$ and place $p_{j}$; I and $O$ denotes input and output places of a transition. For all $i, j$ the values of $n_{i}, t_{j}, t h_{j}, w_{i j}$ lie in the interval $[0,1]$.

Different parameters of the PNN are illustrated in Fig. 1.

\subsection{Encoding of Weights}

Encoded weights are made available by following three basic steps.

\section{Step 1: Transition Updation}

A transition $t r_{j}$ is enabled if all its input places possess tokens (Fig. 2). An enabled transition fires and updates its STT value following the expression

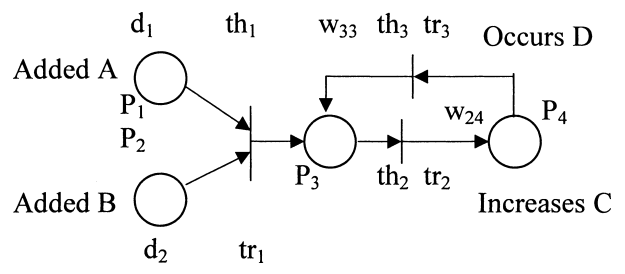

Fig. 1. The architecture of a simple Petri neural network for unsupervised learning.

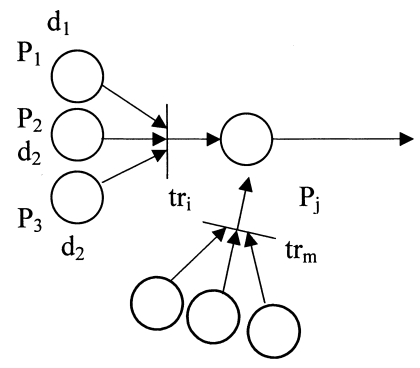

Fig. 2. A Petri neural network showing neuron connectivity.

$$
t_{i}(t+1)=\sqrt[n]{\prod_{j=1}^{n} n_{j}(t)} \wedge u\left[\sqrt[n]{\prod_{j=1}^{n} n_{j}(t)}-t h_{i}\right]
$$

Expression (2) reveals that when $\sqrt[n]{\prod_{j=1}^{n} n_{j}(t)}>t h_{i}$ then

$$
t_{i}(t+1)=\sqrt[n]{\prod_{j=1}^{n} n_{j}(t)}
$$$$
=0 \text {; otherwise }
$$

where $p_{j} \in I\left(t r_{i}\right)$ and $t h_{j}$ denotes the threshold assigned at transition $t r_{i}$.

\section{Step 2: Place Updation}

Place updation in the PNN takes place followed by transition updation, with the help of expression

$$
\begin{aligned}
n_{j}(t+1) & =\sum_{i=1}^{m}\left(t_{i}(t+1) \cdot w_{i j}(t)\right) \\
& =n_{j}(t), \text { when } p_{j} \text { is an axiom }
\end{aligned}
$$

(having no input arc).

where $p_{j} \in O\left(t r_{i}\right)$ and $w_{i j}(t)$ is the weight of the link connected between transition $t r_{i}$ and place $p_{j}$.

Step 3: Weight Updation

Following the transition and place updation process, weight updation finally starts using expression

$$
w_{i j}(t+1)=w_{i j}(t)+t_{i}(t+1) \cdot n_{j}(t+1) .
$$

The above three steps are repeated till an equilibrium in weights are reached in the model. The steady-state value of weights are now adapted in the corresponding links for subsequent reasoning in similar type of problems.

\subsection{The Recall Model}

The encoding and recall process in a cognitive net go in 


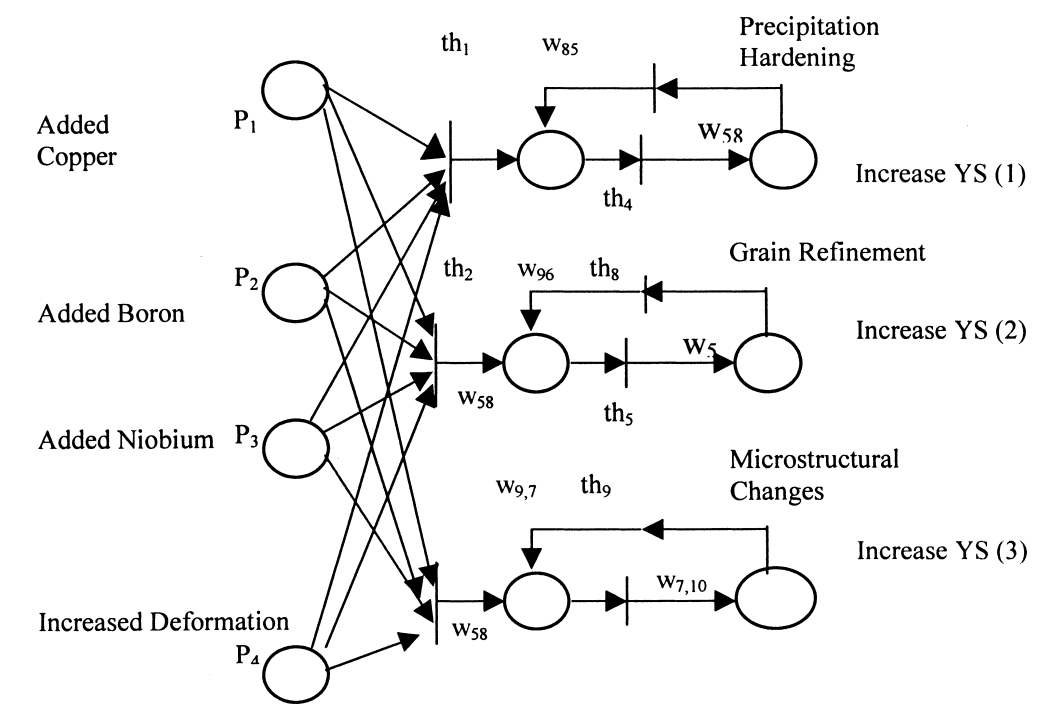

Fig. 3. A PNN describing the effects of copper, niobium, boron and deformation percent on yield strength.

similar way. Once an encoding cycle is over, the recall cycle is executed with the steady-state weights at the links, connected between places and transitions. Initial values of the input places are supplied to the system from the domain expert for analogous problems and revision of values in subsequent places starts. The process of revising the values in different places are terminated after attaining steady-state values mapped at the respective places and given by the expression

$$
\underset{i}{\forall} n_{i}(\dot{t}+1)=n_{i}(\dot{t}) ; \quad \text { at } \dot{t}=\min (t) .
$$

where $\dot{t}$ is called equilibrium time.

\section{The Model}

\subsection{Effects of Copper-Niobium-Boron and Dercent Deformation on the Strength Property}

It is known that copper, niobium and boron improve the yield strength of HSLA steel through precipitation hardening, grain refinement and modifications of microstructure. ${ }^{19)}$ The amount of deformation applied to the steel during hot rolling contributes to the grain refinement and precipitation hardening of the steel. ${ }^{20)}$ In this type of multiphase steels an important contribution to the strength comes from modification of the microstructure, particularly due to the presence of relatively low temperature transformation products of varying morphologies and dislocation density. The amount, composition, morphologies and defect density of these phases are chiefly determined by the nature and amount of alloying elements, as well as the processing parameters. So, in this section, we illustrate a case related to the thermomechanical processing of steels of fixed concentration of carbon, manganese, silicon, nickel, chromium, titanium, molybdenum and fixed slab reheating temperature, finish rolling temperature and cooling rate. In the instant case copper in presence of boron and niobium lowers $\gamma \rightarrow \alpha$ transformation temperature. This leads to the formation of lath martensites of smaller lath width $(\sim 0.1 \mu \mathrm{m})$ upon thermomechanical processing followed by direct quenching.

The following rule bases are developed from the understanding of metallurgy of HSLA steel.

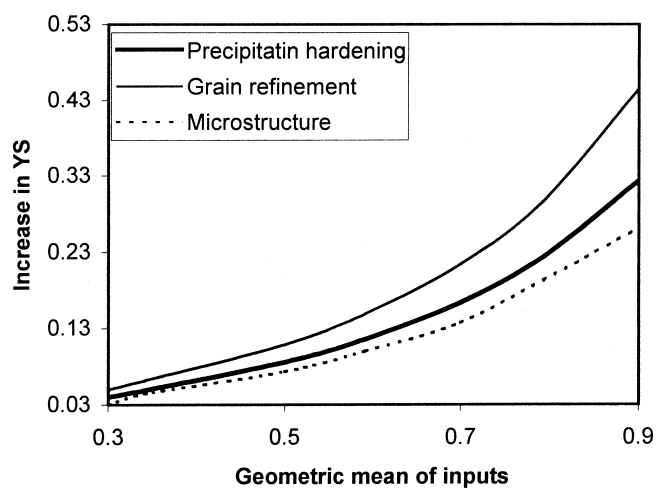

Fig. 4. The prediction values of the network regarding increase in YS for different geometric mean values of the inputs.

1. Added copper, Added boron, Added niobium, Increased deformation - Increases YS (1)

2. Increases YS (1)-Occurs precipitation hardening

3. Added copper, Added boron, Added niobium, Increased deformation - Increases YS (2)

4. Increases YS (2)-Occurs grain refinement

5. Added copper, Added boron, Added niobium Increases YS (3)

6. Increases YS (3)-Occurs microstructural changes

These rules are placed in the network designed to suit the unsupervised learning in PNN (Fig. 3). All values of copper, boron, niobium and deformation percent are presented in normalised condition and are to lie within the 0 tol. The initial belief of each proposition in the PNN for the above case is known. Since among the three mechanisms, grain refinement has the highest toughening effect in a thermomechanically processed HSLA steel, the initial weights (beliefs) set for training are 0.35 for grain refinement, 0.32 for precipitation hardening and 0.30 for microstructural changes. The steady-state weights (CF) are estimated with the help of the encoding model of associative memory realized by PNN. The certainty factors are used for estimating the increase in yield strength due to different strengthening mechanisms. The prediction values of the network regarding the increase in YS for different geometric mean values of the inputs are shown in Fig. 4. 


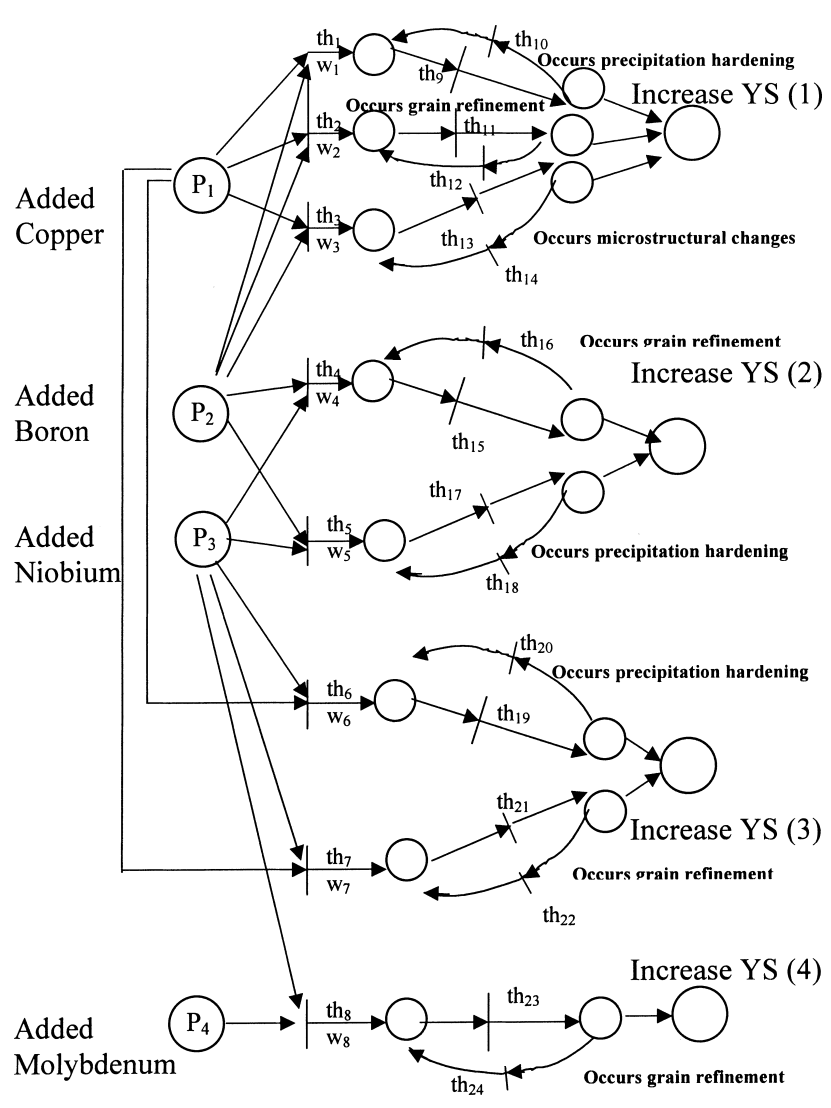

Fig. 5. A PNN describing the effects of copper, niobium, boron and molybdenum on yield strength.

\subsection{Effects of Copper, Niobium, Boron and Molybde- num on Strength Due to Different Mechanisms}

The strengthening effects of copper, niobium and boron are discussed earlier in this section. Molybdenum has an appreciable effect on the yield strength of HSLA steel due to grain refinement. To get a detailed understanding of the strengthening process of steel containing molybdenum along with the other three alloying elements, a more complicated network has been designed (Fig. 5).

The rule bases developed from the prior knowledge of HSLA steel are stated below.

1. Added copper, Added boron - Increases YS $(1,1)$

2. Increases YS $(1,1)$-Occurs precipitation hardening

3. Added copper, Added boron - Increases YS $(1,2)$

4. Increases YS $(1,2)$ - Occurs grain refinement

5. Added copper, Added boron - Increases YS $(1,3)$

6. Increases YS $(1,3)$-Occurs microstructural changes

7. Added niobium, Added boron-Increases YS $(2,1)$

8. Increases YS $(2,1)$ - Occurs grain refinement

9. Added niobium, Added boron-Increases YS (2, 2)

10. Increases YS $(2,2)$-Occurs precipitation hardening

11. Added niobium, Added molybdenum-Increases YS $(3,1)$

12. Increases YS $(3,1)$ - Occurs grain refinement

Alike the previous case, the input values of copper, niobium, boron, and molybdenum are given in normalised condition i.e. within the 0 to 1 . Unlike in the previous case, where some arbitrary values ranging between 0 to 1 have
Table 2. Initial weights assigned to the network describing the effects of copper, niobium, boron and molybdenum on yield strength.

\begin{tabular}{|c|l|c|}
\hline $\begin{array}{c}\text { Alloying } \\
\text { elements }\end{array}$ & \multicolumn{1}{|c|}{ Strengthening mechanism } & Initial weight \\
\hline \multirow{2}{*}{$\mathrm{Cu} \& \mathrm{~B}$} & Precipitation hardening & 0.42 \\
\cline { 2 - 3 } & Grain refinement & 0.32 \\
\cline { 2 - 3 } & Microstructural changes & 0.38 \\
\hline \multirow{2}{*}{$\mathrm{Nb} \& \mathrm{~B}$} & Grain refinement & 0.45 \\
\cline { 2 - 3 } & Precipitation hardening & 0.48 \\
\hline \multirow{2}{*}{$\mathrm{Cu} \& \mathrm{Nb}$} & Precipitation hardening & 0.42 \\
\cline { 2 - 3 } & Grain refinement & 0.38 \\
\hline $\mathrm{Nb} \& \mathrm{Mo}$ & Grain refinement & 0.40 \\
\hline
\end{tabular}

Table 3. Threshold values assigned to the network describing the effects of copper, niobium, boron and molybdenum on yield strength (obtained through ANN 21)).

\begin{tabular}{|c|l|c|c|c|}
\hline $\begin{array}{c}\text { Alloying } \\
\text { elements }\end{array}$ & Strengthening mechanism & $\begin{array}{c}\mathbf{1}^{\text {st }} \\
\text { threshold }\end{array}$ & $\begin{array}{c}\mathbf{2}^{\text {nd }} \\
\text { threshold }\end{array}$ & $\begin{array}{c}\mathbf{3}^{\text {rd }} \\
\text { threshold }\end{array}$ \\
\hline \multirow{2}{*}{$\mathrm{Cu} \& \mathrm{~B}$} & Precipitation hardening & 0.12 & 0.12 & 0.14 \\
\cline { 2 - 5 } & Grain refinement & 0.10 & 0.10 & 0.08 \\
\cline { 2 - 5 } & Microstructural changes & 0.06 & 0.06 & 0.04 \\
\hline \multirow{2}{*}{$\mathrm{Nb} \& \mathrm{~B}$} & Grain refinement & 0.10 & 0.10 & 0.08 \\
\cline { 2 - 5 } & Precipitation hardening & 0.12 & 0.12 & 0.14 \\
\hline \multirow{2}{*}{$\mathrm{Cu} \& \mathrm{Nb}$} & Precipitation hardening & 0.14 & 0.14 & 0.16 \\
\cline { 2 - 5 } & Grain refinement & 0.08 & 0.08 & 0.06 \\
\hline $\mathrm{Nb} \& \mathrm{Mo}$ & Grain refinement & 0.08 & 0.08 & 0.06 \\
\hline
\end{tabular}

been placed as initial weights, the weight factors in this particular exercise are chosen in accordance with the metallurgical understanding of the system (Table 2). The threshold values chosen at different transitions for a successful training are placed in Table 3. The weight factors and transition values chosen for the present network are determined from the trained neural networks successfully predicting similar problems. ${ }^{21)}$ The prediction values of the network regarding the increase in YS for different inputs are shown in Fig. 6.

\section{Discussion}

Though, there may exist one dominant strengthening mechanism, as seen in the above-mentioned cases, it is also possible that several strengthening mechanisms operate in tandem. This stems from the fact that same set of input variables exerts appreciable influence onto more than one strengthening mechanisms. Therefore the effect of different input variables on different strengthening mechanisms is modelled through Petri neural network using unsupervised learning technique. In the first case (Fig. 3), the effects of copper, niobium, boron and deformation percent on the different strengthening mechanisms are predicted. The results (Fig. 4) show that for all values of the inputs the contribution of precipitation hardening in determining the final strength of the steel is maximum. This is followed by grain refinement and microstructural changes. The difference in contribution due to three different strengthening mechanisms is seen to increase with the increase in corresponding input values. Seemingly this is consistent with the metallur- 

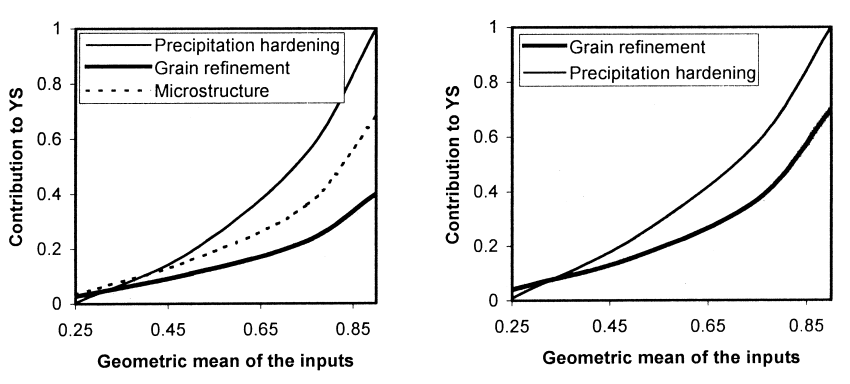

(a)

(b)
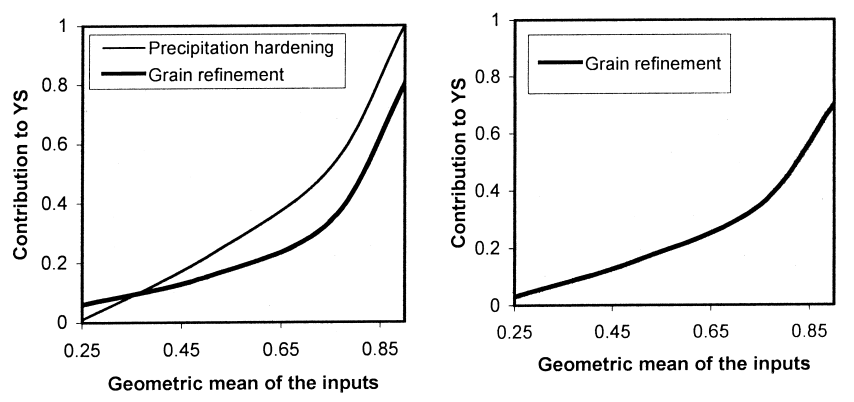

(c)

(d)

Fig. 6. The prediction values of the network regarding increase in YS for the inputs (a) copper and boron, (b) niobium and boron, (c) copper and niobium, and (d) niobium and molybdenum.

gical understanding, because it is already known that the extent of precipitation hardening increases with higher amount of solute atoms in solution as it increases the precipitate density in the microstructure. Thus the slope of increase in strength also becomes higher at higher values of inputs. Grain refinement is equally improved with increasing solute content but it reaches a saturation value quite often. For example, niobium in TMCP steels effects grain refinement by offering more intragranular nucleation sites by way of stabilising dislocation sub-structures. Once the deformation bands are stabilised fully, any extra precipitates of niobium carbide, if present, would not be important for grain refinement, rather, it will continue to enhance precipitation hardening and hence yield strength. The summation of the increases in YS due to different mechanisms was found to be reliable when compared with the experimental results.

In the second model (Fig. 5), the combined effects of the alloying elements on the different strengthening mechanisms are successfully predicted by using suitable weights and threshold values. The weights (Table 2) have been so assigned as to make the predicted values compatible with the metallurgical understanding. In case of addition of copper and niobium, precipitation hardening dominates over grain refinement at higher levels of additions. So the weight assigned for the precipitation hardening due to copper and niobium is higher than that for grain refinement. However, for a solute content less than he solubility limit, one must not expect any contribution from precipitation hardening. Hence the model must recognise a threshold value of inputs below which the model output should yield a zero value. This factor is taken into account through the assignment of suitable threshold values (Table 3), in such a way that when the geometric mean of the normalised values of copper and niobium is less than/equal to 0.25 the network shows zero output against precipitation hardening due to addition of copper-niobium. In Fig. 6, the above fact is beautifully reflected, as it is seen that precipitation hardening has the higher contribution towards strength at higher values of input variables, but for lower values the network ceases to operate. The rates of improvement in strength due to all the three strengthening mechanisms are found to be higher for higher values of inputs. Though not quantified, the output results envisage quantitatively that the final increase strength due to all the input variables is compatible with experimental observations. This is done by finding out the cumulative weightage of different strengthening mechanisms at any two sets of input levels and comparing their ratio with the ratio of experimentally observed yield stresses in either cases.

However one major limitation of this kind of network is that the beliefs in all places may not reach an equilibrium state after sufficient number of cycles. ${ }^{18)}$ In such cases the beliefs may increase or decrease slowly or oscillate. The oscillation in the belief values in this type of networks is known as 'limitcycles'. The 'limitcycles' may be avoided either through addition of more information in the network, or through de-linking the cycles carefully. In the present study the cycles have been de-linked by adjusting the threshold values suitably.

\section{Conclusion}

(1) The effect of input variables on different strengthening mechanisms can be successfully modelled through Petri neural network using unsupervised learning technique.

(2) The combined effects of alloying elements for different strengthening mechanisms can be predicted successfully when weights and threshold values are chosen on the basis of metallurgical understanding. Therefore, such unsupervised learning technique applied through Petri neural network is very effective to create an associative memory of input-output relations in unknown data sets so that the same can be subsequently used as a predictive tool. This entirely new approach is highly efficient in describing the effects of independent variables onto the mechanical properties of steel.

(3) The oscillation in the belief values during training of the Petri neural network can be avoided by delinking the training cycles through suitable adjustment of threshold values.

\section{REFERENCES}

1) S. Ke. Chen and Y. Chang: IEEE Trans. Knowledge Data Eng., 2 (1990), 311.

2) H. K. D. H. Bhadeshia: ISIJ Int., 39 (1999), 966.

3) V. Narayan, R. Abad, B. Lopez, H. K. D. H. Bhadeshia and D. J. C. Mackay: ISIJ Int., 39 (1999), 999.

4) P. Myllykoski: J. Mater. Process. Technol., 79 (1998) 393.

5) Z. Y. Liu, W. D. Wang and W Gao: J. Mater. Process. Technol., 57 (1996), 332.

6) A. Y. Badmos, H. K. D. H. Bhadeshia and D. J. C. McKay: Mater. Sc. Technol., 14 (1998), 1221.

7) L. X. Kong and P. D. Hodgson: ISIJ Int., 39 (1999), 991.

8) S. Datta and M. K. Banerjee: Scand. J. Metall., 33 (2004), 310. 
ISIJ International, Vol. 45 (2005), No. 1

9) A. Konar and A. K. Mondal: IEEE Trans. Knowledge Data Eng., 8 (1996), 96.

10) S. Datta and M. K. Banerjee: ISIJ Int., 44 (2004), 846.

11) B. Kosko: Neural Networks and Fuzzy Systems, Prentice Hall, New Dehli, (1994), 299.

12) W. Reisig: Petri Nets: An Introduction, Springer-Verlag, New York, (1985), 3.

13) J. Sil: Doctoral Thesis, Jadavpur University, (1995).

14) S. Datta, J. Sil and M. K. Banerjee: ISIJ Int., 39 (1999), 986.

15) J. Sil and A. Konar: AMSE Publication, 36 (1995), 1.
16) J. Sil and A. Konar: J. Sci. Ind. Res., 53 (1994), 595.

17) D. O. Hebb: The Organization of Behavior, John Wiley \& Sons, New York, (1949), 62.

18) A. Konar: Artificial Intelligence and Soft Computing, CRC Press, Florida, US, (2000), 697.

19) A. L. Desy: Trans. Iron Steel Inst. Jpn., 14 (1974), 139.

20) I. Tamura, H. Sekine, T. Tanaka and C. Ouchi: Thermomechanical Processing of High Strength Low Alloy Steels, Butterworths, London, (1988), 1.

21) S. Datta: Doctoral Thesis, B. E. College (D.U.), (2003). 